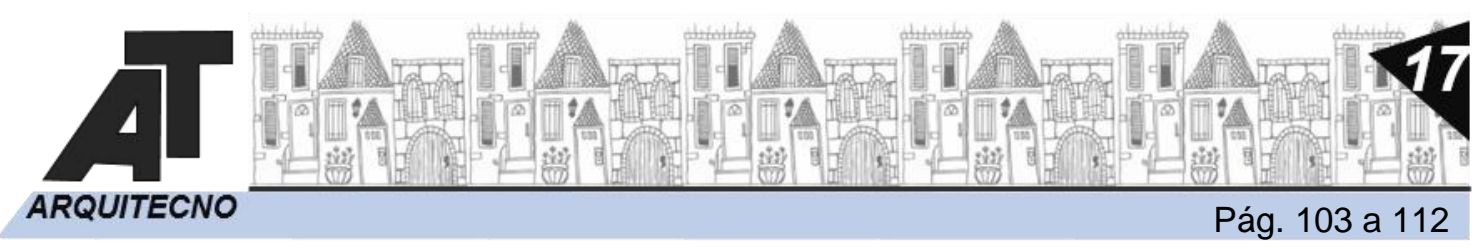

\title{
Matriz de valoración de infraestructura y equipamiento de Terminales terrestres de ciudades intermedias del Ecuador: ciudad de estudio Quevedo
}

\section{Valuation matrix of infrastructure and equipment of land terminals of intermediate cities of Ecuador: city of study Quevedo}

\author{
Cristina Paola Suárez Loor ${ }^{1}$ y Agustin Santiago Vanegas Peña² \\ Maestría en Arquitectura, con mención en Proyectos Arquitectónicos y Urbanos, Facultad de \\ Arquitectura, Universidad San Gregorio de Portoviejo-USGP, Portoviejo, Ecuador.
}

arqui.csuarez@gmail.com; asvanegas@uazuay.edu.ec

\section{RESUMEN}

El presente trabajo aborda la creación de una matriz de valoración para Terminales de transporte terrestres de ciudades intermedias, aplicado a la ciudad de Quevedo en Ecuador, con base en la necesidad de crear una estructura metodológica que evalúe y ponga en consideración las necesidades de la presente terminal. El objetivo general del presente trabajo de investigación fue establecer una matriz de análisis valorativo para determinar la eficacia en la infraestructura y el equipamiento a travéz de una perspectiva humana, definiendo las variables metodológicas, identificando los requerimientos funcionales y aplicando la matriz al caso en cuestión. Como metodología, se valoraron preguntas de investigación, con el planteamiento de variables, categorías e indicadores. Para la recolección de la información se hicieron uso de fichas de observación, entrevistas y encuestas. Los resultados obtenidos se enfocaron en cuanto a las variables como: ciudad, divisiones urbanas, construcción y requerimientos funcionales. Finalmente se concluye, con los resultados presentados en la matriz de valoración.

\section{ABSTRACT}

This work addresses the creation of an assessment matrix for Terrestrial Terminals of intermediate cities, applied to the city of Quevedo in Ecuador, based on the need to create a methodological structure that evaluates and takes into consideration the needs of this terminal. The general objective of this investigation was to establish an analysis matrix evaluation to determine the effectiveness of the infrastructure and equipment through a human perspective, defining the methodological variables, identifying the functional requirements and applying the matrix to the case in question. As a methodology, research questions were assessed, with the approach of variables, categories and indicators. To collect the information, observation files, interviews and surveys were used. The results obtained were focused on variables such as: city, urban divisions, construction and functional requirements. Finally, it was concluded, with the results presented in the valuation matrix.

PALABRAS CLAVE: eficacia, infraestructura, necesidades, perspectiva humana, requerimientos funcionales.

KEY WORDS: effectiveness, infrastructure, needs, human perspective, functional requirements.

FECHA DE RECEPCIÓN: 13/3/2021 | FECHA DE ACEPTACIÓN: 12/5/2021

DOI: http://dx.doi.org/10.30972/arq.0174990 


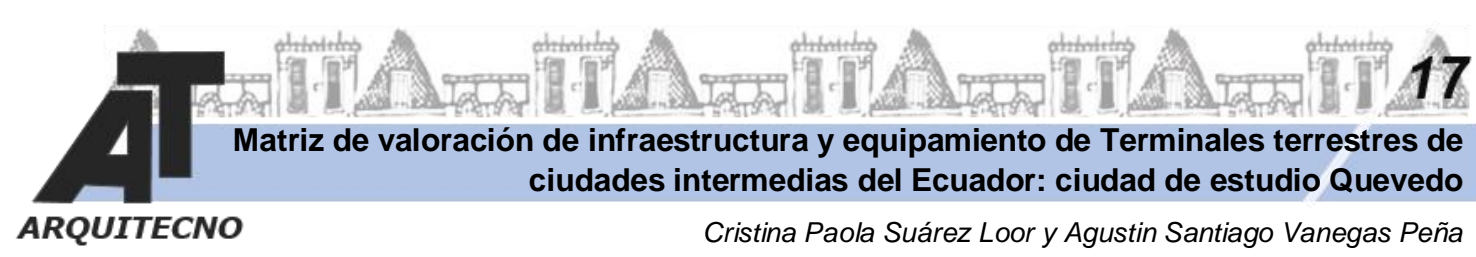

\section{INTRODUCCIÓN}

La presente investigación se toma como referencia, la necesidad de crear una estructura metodológica para establecer un análisis de los requerimientos de las Terminales Terrestres de ciudades intermedias del país, donde se puede identificar variables con sus respectivas categorías e indicadores de calidad y funcionalidad, con el fin de contribuir con el desarrollo vial, vehicular y de crecimiento sostenible de su población, estandarizando lineamientos concretos que ayuden a determinar la eficacia de los terminales terrestres en el país.

En la búsqueda de investigaciones similares no se encuentran estudios específicos sobre algún tipo de metodología para evaluar estos espacios públicos, sin embargo, las Terminales Terrrestres presentan varias problemáticas como: Según (Loaiza, 2016) los problemas en los Terminales Terrestres se clasifican en tres categorías: Ténicos, que se relacionan a la planificación; Funcionales, que abarcan la inexistencia de espacios para el funcionamiento; y Externos; que están relacionados con el entorno.

De esta manera, en la creación de la matriz de valoración para Terminales Terrestres de ciudades intermedias, los problemas se enfocarán en cuatro variables: Ciudad, Divisiones Urbanas, Construcción y Requirimientos funcionales.

(Rojas, 2018) manifiesta que las Terminales Terrestres son consideradas como un espacio físico arquitectónico que permite el desarrollo urbano de las ciudades, brindando un servicio de calidad para los usuarios que necesitan transportarse de un lugar a otro.

La Terminal Terrestre es una infraestructura que debe tener un ambiente de funcionalidad adecuado para recibir a propios y extraños con una atención de calidad, además su diseño permite que se pueda identificar culturalmente la ciudad, mejorando el aspecto urbanistico y calidad de vida del sector donde se ubique.

Para esto la propuesta a realizar, y en este sentido la creación de una matriz que permite conocer la situación actual en el que se encuentran las Terminales Terrestres como su ubicación, accesicibilidad, conectividad, sistema construcctivo, calidad, confort y funcionalidad. En el cantón Quevedo no se han realizado estudios relacionados con el tema, por lo cual se le tomará como caso de estudio para el desarrollo de la presente investigación, que constituye el objetivo final de este trabajo.

\section{METODOLOGIA}

De forma general para el desarrollo de la introducción y el diseño de la Matriz de Valoración se enfocó dentro de la siguiente interrogante: ¿De qué manera las variables metodológicas intervienen en la valoración de los terminales terrestres de ciudades intermedias? Para lo cual se utilizaron mapas y cartografía como distribución clásica, determinándo variables como Ciudad, Divisiones urbanas, Construcción y Requerimientos funcionales.

La recolección de la información se realizó por medio de fichas de observación, entrevistas y encuestas. Dentro de la variable Ciudad se estudiaron categorías como la ubicación, número de habitantes, accesibilidad y conectividad. Mientras que para la variable de Divisiones urbanas se plantearon categorías como Forma urbana, Dinámicas urbanas, Calidad urbana, Economía, Movilidad y Vegetación. Para la variable de Requerimientos funcionales donde se analizaron los espacios de una Terminal Terrestre. Finalmente, para la variable Construcción se analizó el sistema constructivo, los materiales constructivos y los detalles constructivos. 
Ademas, el trabajo de investigación se apoya en la metodología de Análisis de Lugar de Jan Gehl que ha sido puesta en práctica garantizando el éxito en países como México, Chile, Colombia, etc. pretende aproximar al arquitecto/urbanista hacia el territorio, entrando en contacto directo con la gente, con las dinámicas de la ciudad y en esa interacción es capaz de entender la ciudad/sitio, permitiéndole realizar una propuesta con visión y estrategias.

Para la metodología dentro de la Matriz de Valoración se realizó la siguiente interrogante de investigación: ¿Con base a qúe criterios se identificarán los requerimientos funcionales de las Terminales Terrestres de las ciudades intermedias? Para responder a esto se utilizaron planos como distribución clásica, determinándo los requerimientos funcionales como única variable. La recolección de la información se realizó por medio de fichas de observación, entrevistas y encuestas. Para analizar los requerimientos funcionales se estableció como categoría las áreas de la terminal terrestre y como indicadores las diferentes dependencias físicas (existencia, cantidad y funcionalidad).

Mientras que para el análisis de las conclusiones se basó en contestar a la siguiente cuestión: ¿La aplicación de la matriz permitirá realizar un análisis valorativo en las terminales terrestres, para emitir las respectivas conclusiones y recomendaciones? Para lo cual se determinaron las conclusiones a partir del análisis de los apartados anteriores. Dicha matriz pretende aproximar al arquitecto/urbanista hacia el territorio, teniendo un contacto directo con los beneficiados, con las dinámicas de la cuidad, permitiéndole realizar una propuesta con visión y estrategias. Las dinámicas humanas permitirán descubrir como se desempeña el ser humano frente a los espacios públicos, creando estrategias y propuestas para generar la ciudad (Gehl, 2017).

Cabe resaltar que se buscaron otras metodologías de calificación para comparar con esta propuesta y no se encontró una que se ajuste a esta investigación, cada variable, fue estudiada y analizada en una matriz independiente, conjuntamente con sus categorías, indicadores y métodos de análisis, haciéndose énfasis en la observación y la encuesta como herramientas principales.

\section{DESARROLLO}

Debido a los problemas desarrollados en las Terminales Terrestres se constituye una relación de varios aspectos fundamentales para la valoración de los mismos, la creación de la matriz de valoración está conformada por una tabla gráfica donde cada casilla cuenta su variable y estas a su vez con su categoría. Tomando en cuenta a (Jiménez, 2018) una matriz puede ser considerada como una lista de control de dos dimensiones; la una muestra las características del proyecto y la otra se identifica las categorías que pueden ser afectadas por el mismo.

De tal forma, la matriz asigna una calificación cuantitativa a dicha actividad evaluada, y la validez depende de la realcion que se les dé a los criterios de la escala y a los valores asignados a cada una de las valoraciones tomadas en cuenta. La propuesta de la creación de la matriz de valoración cuenta con cuatro variables las cuales van a permitir valorar la calidad del servicio, la funcionabilidad de las Terminales Terrestres en las ciudades intermedias.

\section{Variables}

Estás variables de la matriz van a permitir la valoración de la Terminal Terrestre por eso es necesario el estudio de cada una de ellas, las cuales son: Ciudad, Divisiones urbanas, Construcción y Requerimientos funcionales. 


\section{7}

Matriz de valoración de infraestructura y equipamiento de Terminales terrestres de ciudades intermedias del Ecuador: ciudad de estudio Quevedo

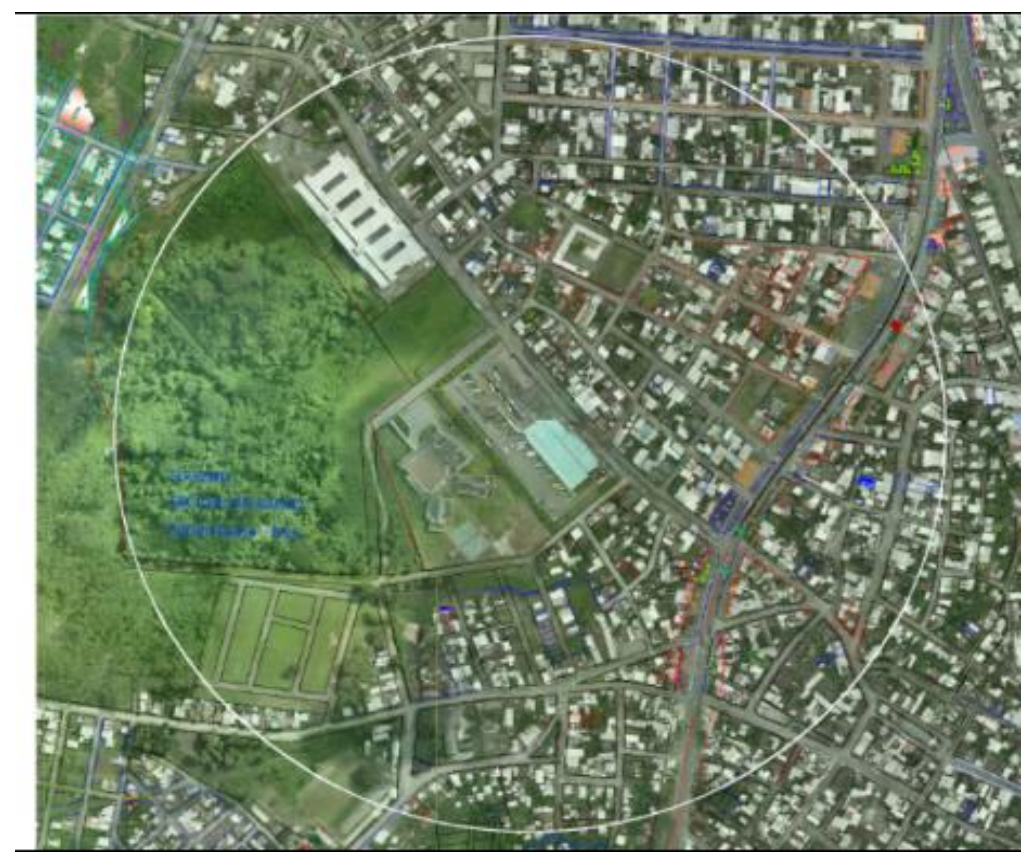

Figura 1. Plano del área del Terminal Terrestre.

Fuente: Google maps

Tabla 1. Matriz de valoración de Terminales Terrestres de ciudades intermedias - Ciudad.

\begin{tabular}{|c|c|c|c|c|c|c|c|c|}
\hline \multicolumn{2}{|c|}{$\begin{array}{l}\text { NOMBRE DE LA TERMINAL: } \\
\text { CIUDAD: } \\
\text { HABITANTES: } \\
\text { \# PASAJEROS DIARIOS: }\end{array}$} & $\begin{array}{l}\text { CIUDAD DE QUEVEDO } \\
\text { QUEVEDO } \\
219.589,74 \text { HAB. } \\
6000 \text { USUARIOS/DÍA } \\
\end{array}$ & & & \multicolumn{4}{|c|}{$\begin{array}{l}\text { CATEGORÍA: } \quad \text { T3 - NO CATEGORIZADO } \\
\text { ÁREA DE TERREN( } 25.000,00 \mathrm{M} 2 \\
\text { \# DE FRECUENCIA } 1.200,00 \text { FRECUENCIAS/DÍA }\end{array}$} \\
\hline \multicolumn{3}{|c|}{ Variables } & & & \multirow{2}{*}{$\begin{array}{l}\text { Método de } \\
\text { análisis }\end{array}$} & & \multirow[b]{2}{*}{ Valor } & \\
\hline & Categoría & Indicador & & & & & & \\
\hline & & Opciones & SI & NO & \multirow{4}{*}{ Observación } & $\% \mathrm{P}$ & & \\
\hline \multirow{17}{*}{ Ciudad } & \multirow{3}{*}{ Ubicación } & Centro & & 0 & & 0,02 & \multirow{3}{*}{$8,00 \%$} & \multirow{17}{*}{$13,50 \%$} \\
\hline & & Periferia & 0 & & & 0,08 & & \\
\hline & & Fuera de la ciudad & & 0 & & 0,15 & & \\
\hline & \multirow{4}{*}{ Accesibilidad } & \begin{tabular}{|l|} 
Opciones \\
\end{tabular} & SI & NO & \multirow{4}{*}{ Observación } & & & \\
\hline & & \begin{tabular}{|l|} 
Principales \\
\end{tabular} & 0 & & & 0,02 & \multirow{3}{*}{$15,00 \%$} & \\
\hline & & Secundarias & 0 & & & 0,15 & & \\
\hline & & Terciarias & & 0 & & 0,08 & & \\
\hline & \multirow{4}{*}{ Conectividad } & \begin{tabular}{|l|} 
Variables \\
\end{tabular} & SI & NO & \multirow{4}{*}{ Observación } & & & \\
\hline & & \begin{tabular}{|l|} 
Parroquias \\
\end{tabular} & 0 & & & 0,02 & \multirow{3}{*}{$10,00 \%$} & \\
\hline & & \begin{tabular}{|l|} 
Cantones \\
\end{tabular} & 0 & & & 0,08 & & \\
\hline & & Regiones & & 0 & & 0,15 & & \\
\hline & \multirow{6}{*}{$\begin{array}{l}\text { Área de } \\
\text { Terreno }\end{array}$} & \begin{tabular}{|l|} 
Variables \\
\end{tabular} & SI & NO & \multirow{6}{*}{ Observación } & & & \\
\hline & & T1 - 73,60 M2 & & 0 & & ruera uetrango & \multirow{5}{*}{$20,00 \%$} & \\
\hline & & T2 - 2.922,00 M2 & & 0 & & de categorla & & \\
\hline & & T3 - $11.094,00 \mathrm{M} 2$ & 0 & & & Dentro del & & \\
\hline & & $\mathrm{T} 4-26.037,00 \mathrm{M} 2$ & & 0 & & rango de & & \\
\hline & & T5 - 34.673,00 M2 & & 0 & & categoría 0,2 & & \\
\hline
\end{tabular}

Fuente: Elaboración propia 


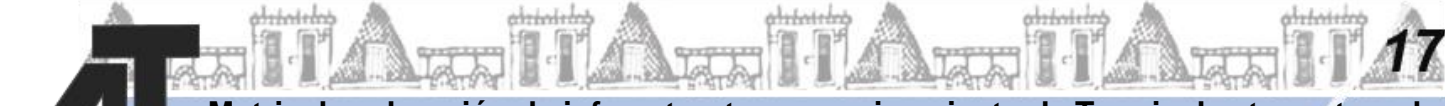 Matriz de valoración de infraestructura y equipamiento de Terminales terrestres de ciudades intermedias del Ecuador: ciudad de estudio Quevedo

Tabla 2. Matriz de valoración de Terminales Terrestres de ciudades intermedias - Divisiones urbanas.

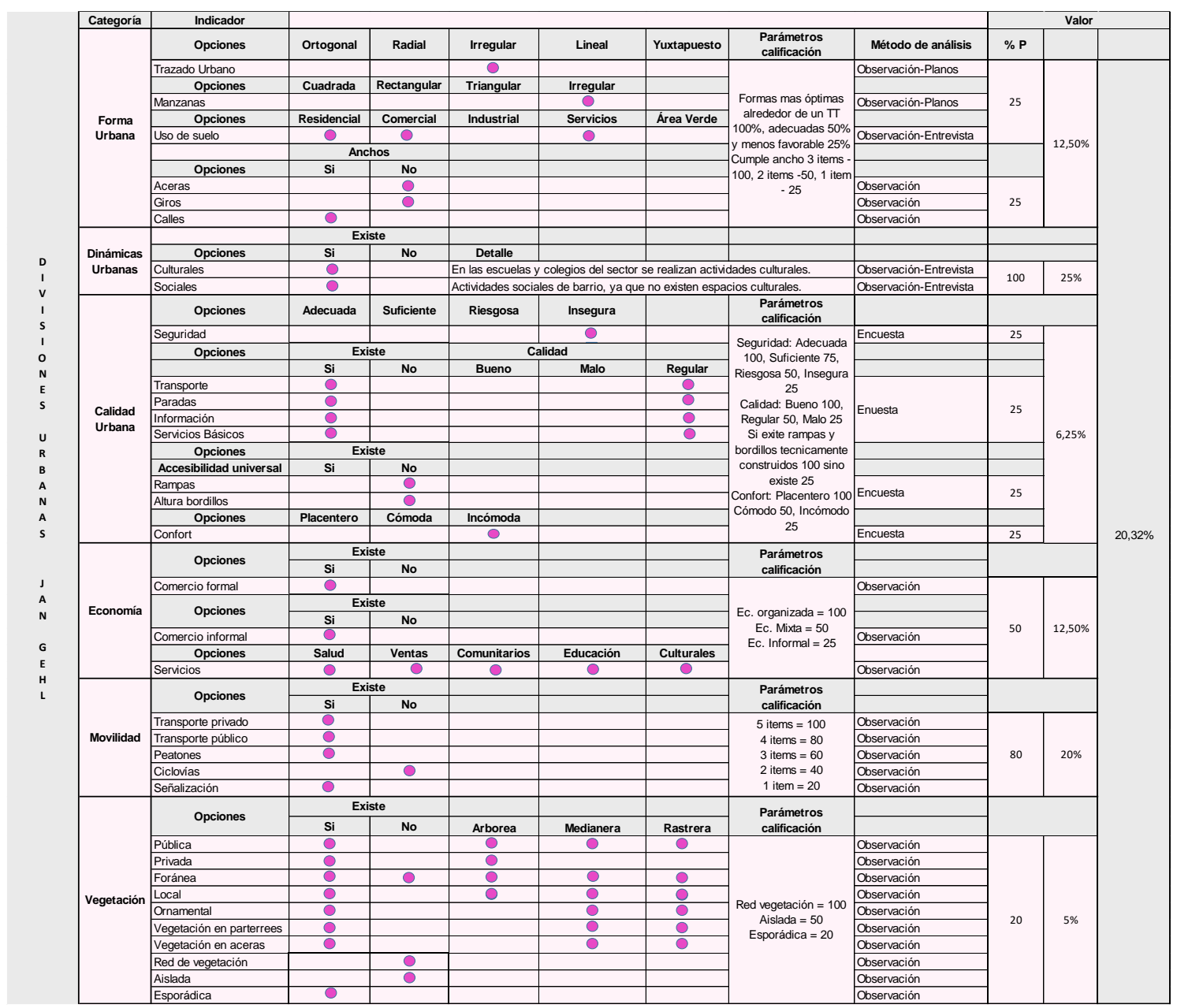

Fuente: Elaboración propia

Tabla 3. Matriz de valoración de Terminales Terrestres de ciudades intermedias - Construcción.

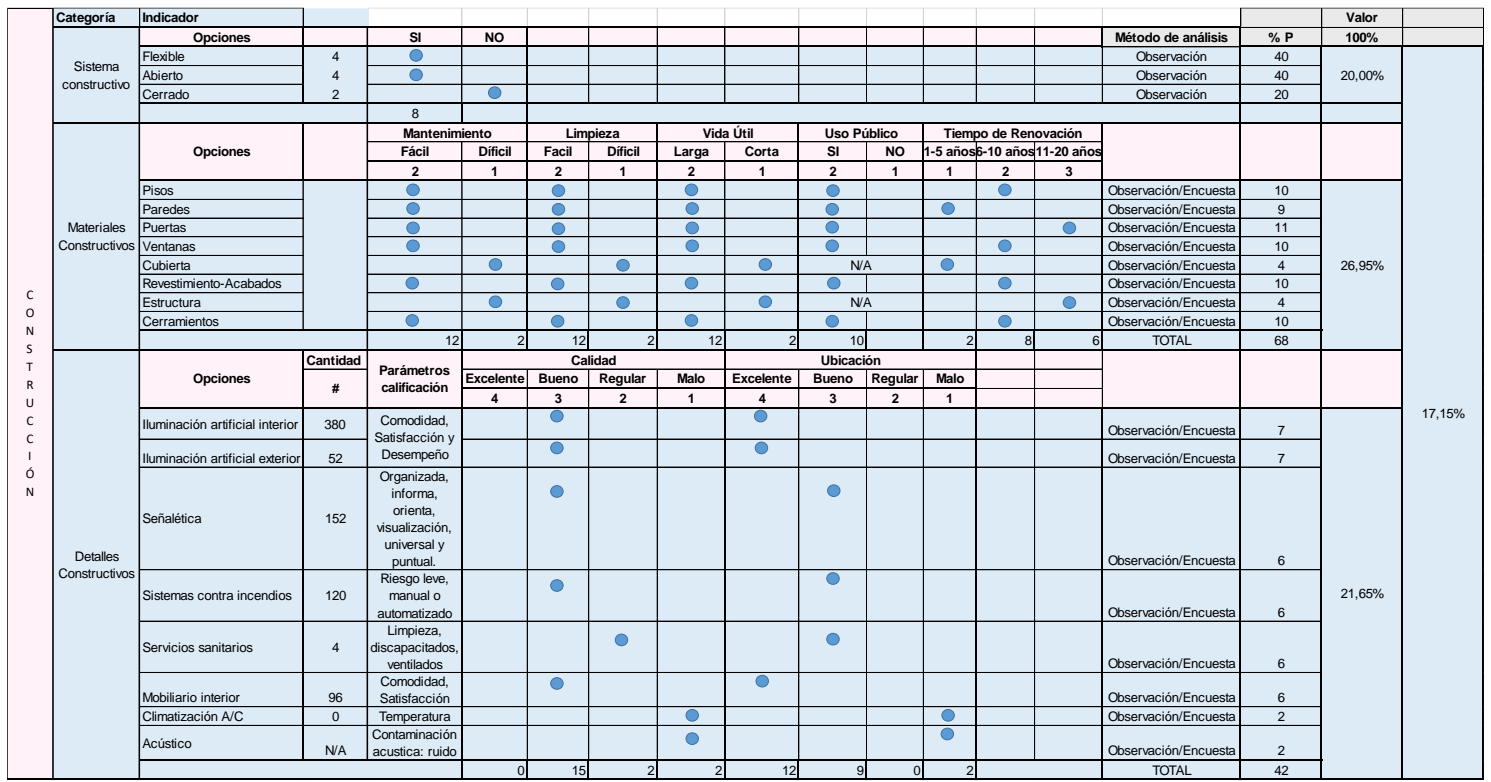

Fuente: Elaboración propia 


\section{7}

Matriz de valoración de infraestructura y equipamiento de Terminales terrestres de ciudades intermedias del Ecuador: ciudad de estudio Quevedo

Cristina Paola Suárez Loor y Agustin Santiago Vanegas Peña

Tabla 4. Matriz de valoración de Terminales Terrestres de ciudades intermedias - Requerimientos funcionales

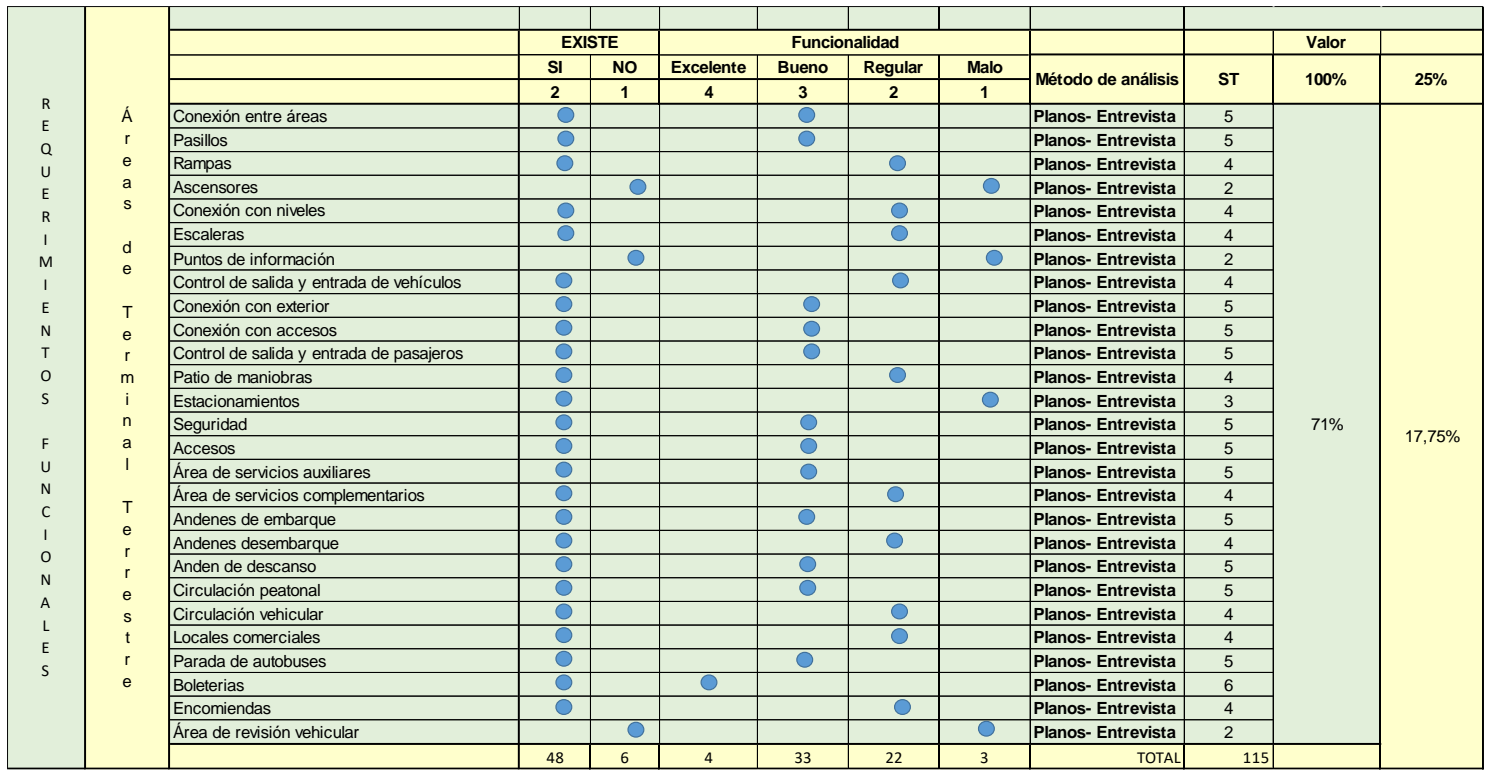

Fuente: Elaboración propia

\section{Discusión de resultados}

Al realizar la investigación acerca de la creación de la Terminal terretre en la cuidad de Quevedo, se pudo conocer que fue inaugurado en el 2002, el primero de la provincia de Los Ríos, antes de la pandemia recibía 6000 usuarios diarios, con un número de 1200 frecuencias por día, debido a la pandemia del Covid-19 la Empresa Pública Quevial administradora de la Terminal terrestre ha reducido su aforo al $50 \%$ por lo que tomaremos los datos antes de la pandemia ya que es el número máximo a quienes sirve la terminal. Esta terminal no está categorizada por la Agencia Nacional de Transito, institución que los regula debido a que tiene que cumplir requisitos de espacios y servicios, gestiones que hoy en día se encuentra realizando la administración.

Para iniciar con la discusión de los resultados es importante recordar las variables mencionadas dentro del presente tema de investigación, las cuales son: Ciudad, Divisiones urbanas, Construcción y Requerimientos funcionales. En el caso de la variable denominada como Ciudad (Tabla 1), se observó que el Terminal terrestre "Ciudad de Quevedo", presenta una ubicación periférica, se accede por medio de una vía principal la E30, esta terminal conecta con rutas hacia ciudades y cantones, los usuarios que necesiten conectarse con regiones deben hacer trasbordo en otras terminales para llegar a su destino, tiene un área de terreno de 2.5 Has y según (Grupo Consultor TTS, 2019) en el cuadro de tipologías de las Terminales Terrestres este se encuentra en la categoría T3.

Para analizar la variable divisiones urbanas (Tabla 2) se determinó un radio de acción de 500 metros alredor de la terminal terrestre, aqui se observó que esta zona tiene varios usos de suelo como residencial, comercial y de servicio, el amanzanamiento es iiregular debido al crecimiento no planificado de la ciudad. Se observaron dinámicas culturales, por medio de actividades de instituciones educativas, además de dinámica sociales por las actividades en las áreas comunales a las que podemos llamar dinámicas de barrio por la falta de espacios culturales, estás se realizan en canchas, calles o aceras. La calidad urbana se pudo analizar a través de encuestas donde se pudo conocer que la zona es insegura debido a que la terminal terrestres se encuentra ubicada en la Parroquia 24 de mayo, existiendo barrios como Galo Plaza y Santa Rosa identificados por la Policia Nacional como zona roja, sin embargo, gozan de servicios básicos con una calidad regular, ya que debido al paso del trasporte público constantemente las tuberías 


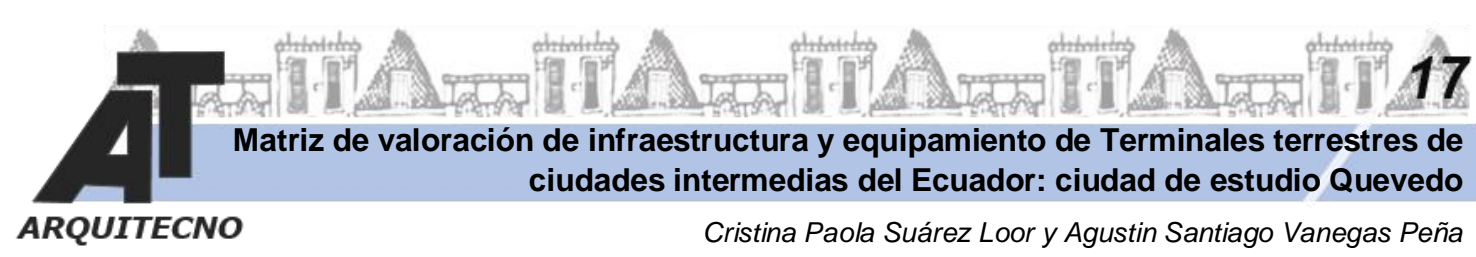

de agua potable se ven afectadas, existe el servicio de transporte público con varias línes de buses, se identifiacaron dos paradas de buses en esta zona una afuera de la terminal terrestre y otra en la via E30. En cuanto a la acceibilidad universal como rampas en aceras y atural de bordillo estás no han sido construido técnicamente y en algunos casos no existen.

Conocer que atrae dinero a la zona lo hicimos a través de la divisiónd Economía, donde el comercio formal e informal se hace presente, al ser un sector populoso existen muchass economías de barrio como tiendas, cybers, talleres mecánicos, destacándose en la calle principal de acceso a la terminal terrestre presencia de infraestructura hotelera ecónmica como como pequeños hoteles y hostales. Esta zona goza también del servicio de salud al estar junto a la terminal el hospital del Instituto Ecuatoriano de Seguridad Social, el segundo hospital mas grande de la provincia, exiten servicios educativos como escuelas fiscales y particulares y la extensión de la Universidad de Loja. Al mencionar a la Movilidad como categoría, se debe indicar el uso del transporte público y privado, la presencia de peatones y la señalización en las vías, no exite ciclovia. Mientras que la Vegetación es existente en la zona, con variantes arbóreas, medianeras y rastreras, entre las que podemos encontrar ficus, palmeras botellas y fénix, guayacan blanco y ornamental, acacias, olivo negro, árbol de tulipan, arboles frutales, durantas, isoras, césped y maní forrajero, pero esás no forman una red de vegetación, sino que se presentan aislada y esporádica.

Para analizar la variable construcción (Tabla 3), se categorizó en primer lugar el sistema constructivo, revisando los planos de la terminal este consta de un sistema abierto ya que su planta está modulada, y su arquitectura es flexible, según (Pinto, 2019) la arquitectura flexible se caracteriza por su adaptibilidad y armonía, y para nuestra investigación es importante conocer si este edificio es flexible para que se puedan realizar recomendaciones. Seguido de los materiales constructivos (pisos, paredes, puertas, entre otros), los cuales son de fácil mantenimiento y limpieza en su mayoría, tienen larga vida útil, de uso público y con prolongados tiempos de renovación, para determinar estas calificaciones se le entrevistó a personal de limpieza y mantenimiento de la terminal.

Concluyendo con los detalles constructivos se muestra como resultados mediante parámetros de calificación midiendo comodidad, satisfacción, desempeño a través de encuestas que estos tienen en su mayoría una calidad regular y su ubicación ha sido desarrollada de una manera excelente y buena, con la excepción de la climatización que no existe pero que Quevial está trabajando para implementarla y realizando estudios de acústica para bajar los niveles a través de asilantes, estudios que tienen gracias al aporte de estudiantes de la Universidad Técnica Estatal de Quevedo.

Los requerimientos funcionales (Tabla 4) existen en su mayoría, al ser un edificio de dos plantas donde en la planta alta funciona la administración no existe acceso para personas con movilidad reducida ya que la única manera de acceder es a través de escaleras, tampoco hay puntos de información y dento de los servicios complementarios no hay una enfermería o de atención inmediata, y tampoco existe un área de revisión vehicular, los buses antes de iniciar su frecuencia hacen sus revisiones en patios propios o en las ecanicas que existen en las calles, sin embargo, la funcionalidad se establece entre buena y regular.

Los rangos calificativos de cada una de las variables permitieron calificar esta terminal terrestre con una puntuación de en la variable Ciudad 13,50/25, Divisiones Urbanas 20,32/25, Construcción 17,15/25 y Requerimientos Funcionales 17,75/25, teniendo un valor total de 68,72/100 (Tabla 5). 
Matriz de valoración de infraestructura y equipamiento de Terminales terrestres de ciudades intermedias del Ecuador: ciudad de estudio Quevedo

Cristina Paola Suárez Loor y Agustin Santiago Vanegas Peña

Tabla 5. Resultados arrojados.

\begin{tabular}{|c|c|c|}
\hline \multicolumn{3}{|c|}{$\begin{array}{l}\text { Matriz de valoración de Terminales Terrestres de ciudades intermedias del Ecuador: ciudad } \\
\text { de estudio Quevedo }\end{array}$} \\
\hline \multirow{4}{*}{ Ciudad (25\%) } & Ubicación & \multirow{4}{*}{$13,50 \%$} \\
\hline & Accesibilidad & \\
\hline & Conectividad & \\
\hline & Área de Terreno & \\
\hline \multirow{6}{*}{ Divisiones Urbanas (25\%) } & Forma Urbana & \multirow{6}{*}{$20,32 \%$} \\
\hline & Dinámicas Urbanas & \\
\hline & Calidad Urbana & \\
\hline & Economía & \\
\hline & Movilidad & \\
\hline & Vegetación & \\
\hline \multirow{3}{*}{ Construcción (25\%) } & Sistema constructivo & \multirow{3}{*}{$17,15 \%$} \\
\hline & $\begin{array}{l}\text { Materiales } \\
\text { Constructivos }\end{array}$ & \\
\hline & Detalles Constructivos & \\
\hline Requerimientos funcionales (25\%) & $\begin{array}{l}\text { Áreas de la Terminal } \\
\text { Terrestre }\end{array}$ & $17,75 \%$ \\
\hline \multicolumn{2}{|l|}{ VALORT } & $68,72 \%$ \\
\hline
\end{tabular}

Fuente: Elaboración propia

\section{CONCLUSIONES Y RECOMENDACIONES}

Cumpliendo con nuestro objetivo se realizó una matriz de análisis valorativo para terminales terrestres, aplicada a Quevedo como ciudad intermedia, para determinar la eficacia en cuanto a su infraestructura y equipamiento desde una perspectiva humana, para realizar esta investigación se determinaron variables y rangos de calificación a través de una investigación exhaustiva y entrevistas a personas experticia en terminales terrestres, a los habitantes de la zona, usuarios, trabajadores, choferes y publico en general.

La variable Ciudad es la que menos puntuación tiene debido a la mala ubicación de la terminal y a la poca conectividad que tiene con regiones siendo Quevedo una ciudad que une a varias, La variable Divisiones urbanas nos permitió conocer problemas de inseguridad que hay en la zona, accesibilidad universal y la falta de una red de vegetación. En la variable Construcción observamos que, aunque la Terminal terrestre tiene un sistema constructivo abierto no tiene mas terreno para ampliar sus áreas de servicios, ya que se encuentra limitado por una Unidad Educativa y un Hospital, los Requerimientos funcionales tienen una funcionalidad en general buena.

Luego de conocer los resultados de la aplicación de la matriz podemos recomendar lo siguiente: Realizar un estudio de conectividad de la terminal con las regiones del país para que la Agencia Nacional de Transito conceda las frecuencias a buses que unan estás regiones, con el fin de mejorar el servicio a los usuarios. La (ANT, 2014) Agencia Nacional de Transito del Ecuador institución que autoriza y aprueba los estudios de una Terminal terrestre menciona en su página web que la ubicación es importante conocerla en la etapa de prediseño para evitar el congestionamiento vehicular en las ciudades y aceptando la recomendación de expertos en Terminales terrestres de nuestro país y pensando en el desarrollo de las ciudades a largo plazo de manera planificada, se sugiere realizar un estudio para el diseño de una nueva terminal terrestre ubicada en las afueras de la ciudad, Realizar un estudio para cambiar de uso las instalaciones de la Terminal Terrestre, solicitar a la Policia Nacional velar por la seguridad de los habitantes en la zona, esto ligado a mejorar la iluminación externa del sitio, el departamento de 


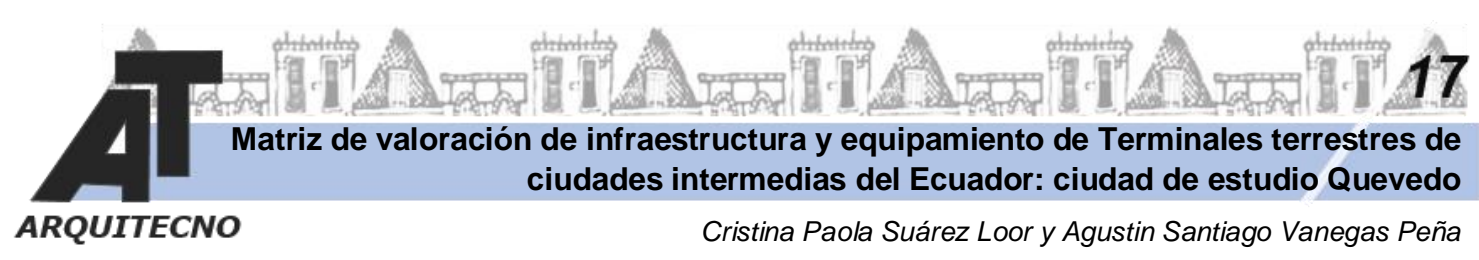

Obras Públicas del Gobierno Autonómo Descentralizado de Quevedo debe hacer un estudio para mejorar la accesibilidad universal de los habitantes de este y todos los sectores de Quevedo, Finalmente se recomienda para la utilización de esta matriz un entranimiento previo para su correcta interpretación y lectura..

Hasta que se realice la consultoría para la nueva Terminal se recomienda mejorar y modernizar las actuales instalaciones de la Terminal terrestre, para mayor comodidad, confort y seguridad de los usuarios. Crear una red verde urbana para integrar sistemáticamente los componentes del entorno natural de la zona.

Esta matriz de evaluación da la pauta para que los funcionarios encargados de la administración de los Terminales Terrestres donde se aplique esta matriz puedan tomar acertadas decisiones en pro del desarrollo sostenible de la ciudad.

\section{BIBLIOGRAFÍA}

ANT, A. N. (2014). www.gob.ec. Obtenido de https://www.gob.ec/ant/tramites/aprobaciontipologia-ubicacion-terminal-terrestre

Capel, H. (1975). "La definición de lo urbano".

Gehl, J. (2014). Cludades para la gente. Buenos Aires: Ediciones Infinito.

Gehl, J. (2017). La Dimensiones humanas en el espacio público. . Chile: Ministerio de Vivienda y Urbanismo.

Grupo Consultor TTS. (2019). "Memoria Técnica Fase 1 Terminal Terrestre del cantón Shushufindi". Shushufindi.

Jiménez, L. (2018). "Las matrices de evaluación: clasificación y normas de forma y fonde para su elaboración". UMBRAL. Obtenido de http://www.colypro.com/ee_uploads/revista/UMBRAL-41.pdf

Loaiza, J. (2016). "http://dspace.utpl.edu.ec/handle/123456789/16306".

Pinto, B. (2019). "Arquitectura y Diseño Flexible". Barcelona.

Rojas, V. (2018).

"https://repositorio.ucv.edu.pe/bitstream/handle/20.500.12692/27355/Roj\%c3\%a1s_VV A.pdf?sequence $=1$ \&isAllowed $=y "$.

Vallejo, F. (2007). "Responsabilidad profesional en la construcción de obras". Derecho del Estado. 